\title{
Análise cladística de Euprepina Hull (Diptera, Bombyliidae, Bombyliinae)
}

\section{Carlos José Einicker Lamas ${ }^{1}$ \\ Márcia Souto Couri ${ }^{1,2}$}

\begin{abstract}
Cladistic analysis of Euprepina Hull, (Diptera, Bombyliidae, Bombyliinae). A cladistic analysis of Euprepina Hull, 1971 (Diptera, Bombyliidae, Bombyliinae), a Neotropical genus that includes ten species, was made. The cladogram was obtained from eight studied species, based on a data matrix with 21 characters, using the program Hennig86. Character states were polarized following outgroup analysis, and an hypothetical ancestor was included in the analysis in order to root the tree. The options used, "ie" and "xs w", resulted in four most parsimonious trees with $\mathrm{ci}=79$, $\mathrm{ri}=80$ and length 115. The monophiletism of Euprepina was supported by two synapomorphies.
\end{abstract}

KEY WORDS. Diptera, Euprepina, Bombyliidae, Bombyliinae, cladistic analysis

Euprepina Hull, 1971, inclui dez espécies conhecidas. Pertence aos Bombyliinae, maior subfamília de Bombyliidae, com 63 gêneros, aproximadamente 1000 nomes específicos válidos, e com ocorrência em todas as regiões biogeográficas (YEATES 1994).

HuLL (1973) propôs 11 tribos para os Bombyliinae: Bombyliini, Acrophthalmydini, Eclimini, Paratoxophorini, Conophorini, Corsomyzini, Mariobezziini, Cythereini, Heterostylini, Crocidiini e Dischistini.

BowDEN (1985) reduziu para oito o número de tribos de Bombyliinae. Eclimini e Cythereini foram elevadas a categoria de subfamília e, Mariobezzini foi incluída na nova subfamília Cythereinae.

EvENHUIS (1991) reconheceu apenas cinco tribos dentre os Bombyliinae: Acrophthalmydini, Bombyliini, Conophorini, Crocidiini, e Dischistini, e posicionou Euprepina nesta última.

YEATES (1994) propôs uma classificação para os Bombyliidae, utilizando análise cladística, tendo reconhecido como tribos de Bombyliinae: Acrophthalmydini, Bombyliini, Conophorini e Eclimini. A até então sub-família Ecliminae apareceu como um dos membros mais distais de Bombyliinae, e por isso recebeu "status" de tribo. Já os Crocidinii, posicionaram-se fora de Bombyliinae e por isso foram considerados como uma subfamília distinta. O autor estabeleceu ainda que os "Dischistini" são parafiléticos, podendo ser tratados como tribo apenas por conveniência taxonômica, até que um estudo mais detalhado sobre as relações dos gêneros de "Dischistini" com os Bombyliini e Eclimini, seja realizado.

1) Museu Nacional. Quinta da Boa Vista, São Cristóvão, 20940-040 Rio de Janeiro, Rio de Janeiro, Brasil.

2) Bolsista do CNPq. 
LAMAS \& COURI (1998) acrescentaram uma espécie nova ao gênero - $E$. beckeri, considerando-a morfologicamente similar a E. truxalia Hull, 1971, por serem as únicas espécies do gênero com labelo longo.

LAMAS \& COURI (1999) revisaram o gênero Euprepina Hull, 1971 e redescreveram oito espécies, apresentaram uma chave para segregação destas espécies, e sinonimizaram E. bicincta Hull, 1971 e E. shannoni Hull, 1971, respectivamente com E. bicincta (Wiedemann), 1830 e E. nuda Hull, 1971. Como resultado, foram consideradas válidas as seguintes espécies: E. nuda Hull, 1971; E. amabilis (Wulp, 1881); E. aperta (Macquart, 1847); E. beckeri Lamas \& Couri, 1998; E. bicincta (Wiedemann, 1830); E. caminaria (Wiedemann, 1830); E. goyaz (Macquart, 1840); E. knutsoni Hull, 1971; E. maracajula Hull, 1971 e E. truxalia Hull, 1971.

Devido a superficialidade das descrições originais e impossibilidade de examinar exemplares de E. aperta e E. goyaz, estas duas espécies não foram incluídas na análise.

\section{MÉTODOS}

A metodologia empregada neste estudo para formular uma hipótese filogenética foi a análise cladística (HENNIG 1966; WILEY 1981). O relacionamento entre as espécies de Euprepina foi obtido através da codificação e análise de 21 caracteres.

Para polarização dos caracteres foi utilizado o método da comparação com grupo-externo (WATROUS \& WHEELER 1981; MADDISON et al. 1984). Utilizou-se para esta comparação outro Dischistini, Heterostylum hirsutum Thunberg, 1827, e duas outras espécies, (Sparnopolius confusus Wiedemann, 1824 e Conophorus fallax Greene, 1921) de uma tribo mais basal, Conophorini. Este grupo, segundo YEATES (1994), seria grupo-irmão de todos os demais Bombyliinae, reunindo condições supostamente plesiomórficas.

Não existe, até a presente data, nenhuma hipótese filogenética para os gêneros de Bombyliinae, tampouco dispunha-se de exemplares de todos os táxons para uma melhor compreensão da diversidade morfológica da subfamília. Por esta razão, preferiu-se criar um ancestral hipotético (MADDISON et al. 1984).

O enraizamento do cladograma, a partir destes três gêneros, representa uma primeira tentativa de entendimento das séries de transformações de caracteres dentro da subfamília, correspondendo assim a uma primeira hipótese de trabalho que poderá ser testada posteriormente com a adição de novos táxons.

Todos os caracteres utilizados são binários, à exceção do caráter 9 que é multiestado, e este juntamente com o caráter 15 (não comparável nos grupos externos), foi tratado como não ordenado "a priori".

Os caracteres foram discutidos na ordem em que aparecem na matriz de caracteres (Tab. I). A codificação "0" representa a condição plesiomórfica do caráter, " 1 " e " 2 " as condições apomórficas. No grupo interno o símbolo "?" foi utilizado quando o caráter não foi examinado e, no grupo externo, quando o caráter não é comparável com os representantes deste grupo.

Para análise de parcimônia, foi utilizado o programa Hennig86 desenvolvido por FARRIS (1988), versão 1.5. Foi utilizado ainda, o programa "Tree Gardener", versão 2.2 (RAMOS 1997), que em interação com o Hennig86, apresenta os dados de forma simplificada. 
$\mathrm{Na}$ análise foram utilizadas as opções "ie*" e "xs w". As séries de transformação obtidas foram submetidas à ponderação sucessiva, otimizando a informação fornecida na matriz, através de pesagem "a posteriori" dos caracteres congruentes (FARRIS 1969; CARPENTER 1988; GOLOBOFF 1993, 1995).

A terminologia empregada foi a de AMORIM (1997).

Tabela I. Matriz de caracteres com a distribuição de 21 caracteres nas espécies de Euprepina.

\begin{tabular}{lllllllllllllllllllllll}
\hline \multicolumn{1}{c}{ Táxons } & 1 & 2 & 3 & 4 & 5 & 6 & 7 & 8 & 9 & 10 & 11 & 12 & 13 & 14 & 15 & 16 & 17 & 18 & 19 & 20 & 21 \\
\hline Ancestral hipotético & 0 & 0 & 0 & 0 & 0 & 0 & 0 & 0 & 0 & 0 & 0 & 0 & 0 & 0 & $?$ & 0 & 0 & 0 & 0 & 0 & 0 \\
Euprepina beckeri & 1 & 0 & 1 & 0 & 1 & 1 & 0 & 1 & 0 & 1 & 1 & 0 & 1 & 1 & 1 & 0 & 0 & 1 & 0 & 0 & 1 \\
Euprepina bicincta & 1 & 0 & 0 & 1 & 1 & 1 & 1 & 0 & 1 & 1 & 1 & 1 & 1 & 0 & 1 & 0 & 1 & 0 & 0 & 0 & 0 \\
Euprepina caminaria & 1 & 1 & 0 & 0 & 0 & 1 & 1 & 0 & 0 & 1 & 1 & 1 & 1 & 1 & 1 & 0 & 0 & 1 & 0 & $?$ & $?$ \\
Euprepina truxalia & 0 & 1 & 1 & 1 & 0 & 1 & 1 & 1 & 2 & 0 & 0 & 0 & 1 & 1 & 1 & 0 & 0 & 0 & 1 & 0 & 1 \\
Euprepina amabilis & 0 & 0 & 0 & 0 & 0 & 1 & 1 & 1 & 1 & 0 & 1 & 0 & 1 & 1 & 0 & 0 & 1 & $?$ & $?$ & $?$ & $?$ \\
Euprepina knutsoni & 0 & 0 & 0 & 1 & 1 & 1 & 0 & 0 & 0 & 1 & 1 & 0 & 1 & 1 & 0 & 0 & 1 & 1 & 1 & 1 & 0 \\
Euprepina maracajula & 0 & 0 & 0 & 1 & 1 & 1 & 0 & 1 & 0 & 0 & 0 & 0 & 1 & 1 & 1 & 1 & 0 & $?$ & $?$ & $?$ & $?$ \\
Euprepina nuda & 0 & 0 & 0 & 1 & 1 & 1 & 0 & 1 & 0 & 0 & 0 & 0 & 1 & 0 & 0 & 1 & 0 & 0 & 1 & 1 & 0 \\
\hline
\end{tabular}

\section{ANÁLISE CLADÍSTICA}

\section{Caracteres e estados}

Caráter 1. Comprimento relativo das cerdas do pedicelo: $($ peso $=10$, passos $=1$, $\mathrm{ci}=100, \mathrm{ri}=100)-(0)$ uniforme; (1) desigual.

A presença de cerdas com comprimento desigual no pedicelo é sinapomorfia para o grupo de espécies E. beckeri, E. bicincta e E. caminaria.

Caráter 2. Comprimento do escapo em relação ao pedicelo: $($ peso $=0$, passos $=2$, $\mathrm{ci}=50, \mathrm{ri}=0)-(0)$ o dobro ou mais que o dobro; (1) menos que o dobro.

$\mathrm{O}$ estado apomórfico ocorre paralelamente em E. caminaria e E. truxalia.

Caráter 3. Comprimento do labelo: (peso $=0$, passos $=2, \mathrm{ci}=50, \mathrm{ri}=0)-(0)$ curto;

(1) longo.

O estado apomórfico ocorre paralelamente em E. beckeri e E. truxalia.

Caráter 4. Cor dos palpos: (peso $=1$, passos $=3, \mathrm{ci}=33$, ri $=33$ ) $-(0)$ uniforme; (1) com duas tonalidades.

Palpos castanhos na metade apical e amarelos na metade basal é apomorfia compartilhada por E. truxalia, E. knutsoni, E. maracajula e E. nuda, com reversão em $E$. amabilis. Ocorre independentente em $E$. bicincta.

Caráter 5. Cor das cerdas no palpo: (peso $=0$, passos $=4, \mathrm{ci}=25, \mathrm{ri}=0)-(0)$ uniforme; (1) com duas tonalidades.

O estado apomórfico é encontrado em quase todas as espécies de Euprepina, com exceção de E. truxalia. Reversões são observadas em E. caminaria e $E$. amabilis.

Caráter 6. Número de segmentos do palpo: (peso $=10$, passos $=1, \mathrm{ci}=100, \mathrm{ri}=$ $100)$ - (0) um segmento; (1) dois segmentos.

Sinapomorfia para Euprepina.

Caráter 7. Comprimento relativo dos pêlos do triângulo ocelar: (peso $=1$, passos $=3, \mathrm{ci}=33, \mathrm{ri}=33)-(0)$ uniforme; (1) desigual. 
Apomorfia compartilhada entre E. bicincta e E. caminaria e aparece paralelamente em E. truxalia e E. amabilis.

Caráter 8. Região do triângulo ocelar: (peso $=1$, passos $=3, c i=33, r i=33)-(0)$ com cerdas esparsas; (1) com tufo de cerdas.

Apomorfia compartilhada por E. truxalia, E. amabilis, E. maracajula e E. nuda, com reversão em $E$. knutsoni e aparecendo paralelamente em $E$. beckeri.

Caráter 9. Número de cerdas notopleurais: (peso $=0$, passos $=33, \mathrm{ci}=66, \mathrm{ri}=0$ ) - (0) 3 ; (1) 4 ; (2) 5 .

A presença de quatro cerdas notopleurais ocorre independentemente em $E$. amabilis e E. bicincta. A ocorrência de cinco cerdas notopleurais consiste em autapomorfia para E. truxalia.

Caráter 10. Calo pós-alar com cerdas negras: $($ peso $=3$, passos $=2, \mathrm{ci}=50, \mathrm{ri}=$ $66)$ - (0) ausentes; (1) presentes.

Apomorfia compartilhada por E. beckeri, E. bicincta e E. caminaria e aparece paralelamente em E. knutsoni.

Caráter 11. Margem posterior do escutelo com cerdas negras: $($ peso $=3$, passos $=$ 2 , $\mathrm{ci}=50, \mathrm{ri}=66)-(0)$ ausentes; (1) presentes.

Apomorfia compartilhada por E. beckeri, E. bicincta, E. caminaria, E. amabilis e E. knutsoni.

Caráter 12. Cor do capítulo do halter: (peso $=10$, passos $=1, \mathrm{ci}=100, \mathrm{ri}=100)-$ (0) amarelo; (1) castanho.

A presença de halter com capítulo castanho é sinapomorfia para E. bicincta e E. caminaria.

Caráter 13. Posição da veia transversal $\mathrm{r}-\mathrm{m}$ em relação à célula discal: (peso $=10$, passos $=1, \mathrm{ci}=100, \mathrm{ri}=100)-(0)$ aquém da metade; (1) além da metade.

Sinapomorfia para Euprepina.

Caráter 14. Comprimento da abertura da célula anal em relação à $r-m$ : (peso $=0$, passos $=3, \mathrm{ci}=33, \mathrm{ri}=0)-(0)$ igual; (1) menor.

Célula anal com abertura menor que o comprimento da veia transversal $\mathrm{r}-\mathrm{m}$ ocorre em todas as espécies de Euprepina, sofrendo reversão apenas em $E$. bicincta e E. nuda.

Caráter 15. Posição da r-m em relação à célula discal: (peso $=10$, passos $=2, \mathrm{ci}=$ $50, \mathrm{ri}=50)-(0) 1 / 4$ distal; (1) $1 / 3$ distal.

Veia transversal $\mathrm{r}-\mathrm{m}$ posicionada no $1 / 3$ distal da célula discal ocorre em todas as espécies de Euprepina, sofrendo reversão em E. nuda, E. amabilis e E. knutsoni.

Caráter 16. Largura da base do abdômen em relação ao ápice: (peso $=10$, passos $=1, \mathrm{ci}=100, \mathrm{ri}=100)-(0)$ ápice muito mais estreito; (1) semelhante.

O estado apomórfico é sinapomorfia para E. maracajula e E. nuda.

Caráter 17. Tergito abdominal II nos machos com faixa branca: (peso $=2$, passos $=2, \mathrm{ci}=50, \mathrm{ri}=50)-(0)$ ausente; (1) presente.

A presença de faixa branca no tergito abdominal II nos machos é apomorfia compartilhada por E. amabilis e E. knutsoni, aparecendo paralelamente em $E$. bicincta. 
Caráter 18. Comprimento do apódema lateral do edeago: (peso $=0$, passos $=3$, ci $=33, \mathrm{ri}=0)-(0)$ curto, não ultrapassando a margem da gonocoxa; (1) longo, ultrapassando a margem da gonocoxa.

O estado apomórfico aparece paralelamente em, E. beckeri, E. caminaria e E. knutsoni.

Caráter 19. Comprimento do distifalo: (peso $=10$, passos $=1, \mathrm{ci}=100, \mathrm{ri}=100)$ - (0) longo, ultrapassando o ápice do lobo do gonocoxito; (1) curto, não ultrapassando o ápice do lobo do gonocoxito.

Distifalo curto, não ultrapassando o ápice do lobo do gonocoxito, é sinapomorfia para o grupo de espécies formado por E. truxalia, E. amabilis, E. knutsoni, E. maracajula e E. nuda.

Caráter 20. Relação comprimento $x$ largura da espermateca: $($ peso $=10$, passos $=$ $1, \mathrm{ci}=100, \mathrm{ri}=100)-(0)$ comprimento igual ao dobro ou menos que a largura; (1) comprimento igual ao triplo da largura.

O estado apomórfico é sinapomorfia para o grupo formado por $E$. truxalia, $E$. amabilis, E. knutsoni, E. maracajula e E. nuda.

Caráter 21. Membrana na base da espermateca: (peso $=0, \operatorname{passos}=2, \mathrm{ci}=50, \mathrm{ri}=$ $0)$ - (0) ausente; (1) presente.

A presença de membrana na base da espermateca aparece independentemente em E. beckeri e E. truxalia.

\section{RESULTADOS E DISCUSSÃO}

A análise inicial dos 21 caracteres, resultou na obtenção de 19 árvores mais parcimoniosas com 43 passos de comprimento, índice de consistência (ci) de 51 e índice de retenção (ri) de 43 .

Com a utilização da ponderação sucessiva, foram obtidas quatro árvores mais parcimoniosas $($ comprimento $=115, \mathrm{ci}=79$ e ri $=80$ ). A organização das espécies em dois grupos foi idêntica nas quatro árvores. A escolhida para discussão (Fig. 1), é a que apresentou melhor resolução com relação ao posicionamento de sinapomorfias para sustentação dos grupos. A árvore de consenso não foi aqui discutida pois ela, embora apresentasse arranjo idêntico dos grupos, apresentava politomia na resolução das espécies de um dos grupos.

O monofiletismo de Euprepina Hull é sustentado, na presente análise, por duas sinapomorfias: palpos com dois segmentos (caráter 6.1) e veia transversal r-m posicionada além da metade apical da célula discal (caráter 13.1).

A topologia do cladograma escolhido demonstra claramente a divisão de Euprepina em dois grupos monofiléticos, um deles possuindo três e o outro quatro táxons terminais. O primeiro grupo formado por E. beckeri, E. bicincta e $E$. caminaria, é sustentado por uma sinapomorfia: pedicelo com cerdas de comprimento desigual (caráter 1.1). O segundo grupo formado por E. knutsoni, E. amabilis, $E$. maracajula, E. nuda e E. truxalia, é sustentado por duas sinapomorfias: distifalo curto (caráter 19.1) e comprimento da espermateca igual ao triplo de sua largura (caráter 20.1).

Para os demais agrupamentos, foram detectadas sinapomorfias para $E$. bicincta + E. caminaria (caráter 12.1) e E. maracajula + E. nuda (caráter 16.1). 


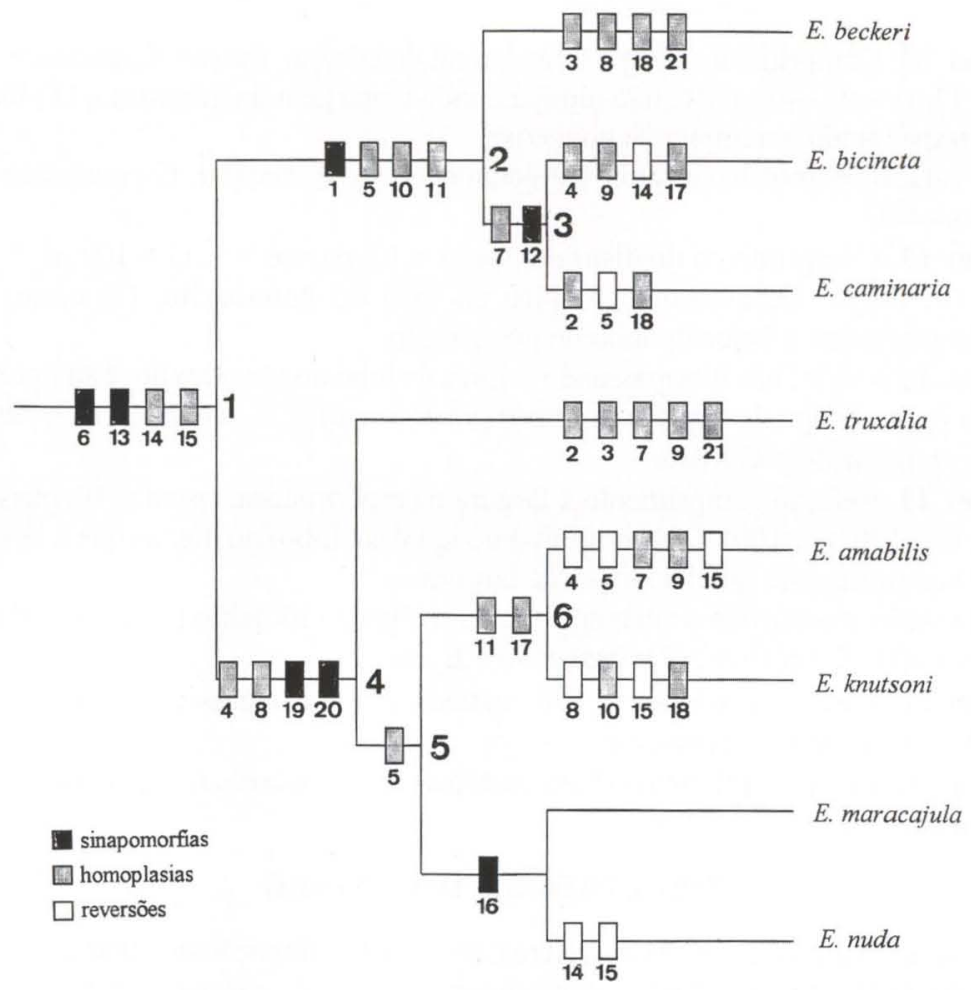

Fig. 1. Cladograma para as espécies de Euprepina com base na análise de 21 caracteres. Comprimento 115, indice de consistência (ci) 79, indice de retenção (ri) 80 .

AGRADECIMENTOS. Ao Dr. Hussan Zaher (Universidade de São Paulo, São Paulo, Brasil) pelas numerosas sugestões e esclarecimentos. Ao Dr. Brian Brown (Natural History Museum of Los Angeles County, Los Angeles, EUA), pelo auxílio na localização do material tipo de Euprepina truxalia Hull, 1971. Aos doutores: Neal Evenhuis (Bernice P. Bishop Museum, Honolulu, Hawaii), David K. Yeates (The University of Queensland, Queensland, Australia), B. Brugge (Instituut voor Taxonomische Zoologie, Amsterdã, Holanda), J.M. Cumming (Canadian National Collection, Ontário, Canadá), Ruth Contreras-Lichtenberg (Naturhistorisches Museum Wien, Viena, Áustria) e ao Dr. José Henrique Guimarães (Museu de Zoologia, Universidade de São Paulo, São Paulo, Brasil), pelo empréstimo de material tipo e outros espécimens.

\section{REFERÊNCIAS BIBLIOGRÁFICAS}

AMORIM, D.S. 1997. Elementos básicos de sistemática filogenética. São Paulo, Sociedade Brasileira de Entomologia, 276p.

BOWDEN, J. 1985. The tribal classification of the Bombyliinae with particular reference to the Bombyliini and Dischistini, and the description of a new genus from South America (Dipt., Bombyliidae). Entomol. Mon. Mag. 121: 99-107. 
CARPENTER, J.M. 1988. Choosing Among Multiple Equally Parsimonious Cladograms. Cladistics 4: 291-296.

EVENHUIS, N.L. 1991. World catalog of the genus-group names of bee flies (Diptera: Bombyliidae). Bishop Mus. Bull. Entomol. 5: 1-105.

FARRIS, J. 1969. A successive approximations approach to character weighting. Syst. Zool. 18: 374-385.

. 1988. Hennig 86, Version 1.5. New York, Published by the author, Port Jefferson Station.

Goloboff, P.A. 1993. Estimating character weights during tree search. Cladistics 9: 83-91.

11: $91-104$.

HenNIG, W. 1966. Phylogenetic systematics. University of Illinois Press, Urbana. HULL, F.M. 1973. The beeflies of the world. The genera of the family Bombyliidae. Bull. U.S. Nat. Mus. 286: 3-687.

LAMAS, C.J.E. \& M.S. CouRI. 1998. A new species of Euprepina Hull, 1971 from Bahia, Brazil (Diptera: Bombyliidae: Bombyliinae). Revta bras. Ent. 41 (2-4): 447-449.

1999. Revisão de Euprepina Hull (Diptera, Bombyliidae, Bombyliinae). Revta bras. Zool. 16 (2): 461-482.

MAdDison, W.P.; M.J. DonOghuE \& D.R. MADDIson. 1984. Outgroup analysis and parsimony. Syst. Zool. 33 (1): 83-103.

RAMOS, T.C. 1997. Tree gardener 2.2. São Paulo, Privately distributed by Thiago Courrol Ramos, Museu de Zoologia, Universidade de São Paulo.

WATROUS, L.E. \& Q.D. WhEELER. 1981. The outgroup comparision method of character analysis. Syst. Zool. 30 (1): 1-11.

WiLEY, E.O. 1981. Phylogenetics. The theory and practice of phylogenetic systematics. New York, John Wiley \& Sons, 439p.

YEATES, D.K. 1994. The cladistic and classification of the Bombyliidae (Diptera: Asiloidea). Bull. Amer. Mus. Nat. Hist. 219: 1-191.

Recebido em 18.VI.1998; aceito em 05.XI.1999. 